\title{
Application Value of lodixanol in Detecting Aortic Dissection of Elderly Patients in Low Dose Scanning Scheme by Idose
}

\author{
Wenfu Yan \\ Department of Radiology \\ the Third Affiliated Hospital of Qiqihar Medical \\ University \\ Qiqihar ,China \\ ywf112915@126.com
}

\author{
Wei Zhao * \\ Department of Radiology \\ the Third Affiliated Hospital of Qiqihar Medical \\ University \\ Qiqihar ,China \\ zhaowei121415@163.com \\ * Corresponding Author
}

Keywords: Aortic dissection; Tomography; CAT Scan; Contrast agent; Radiation dose

\begin{abstract}
Objective To investigate the application value of combined application of reducing the radiation dose by iDose low-dose scan strategy and using iodixanol as contrast agents in elderly CTA detection to aortic dissection. Methods 36 cases of aortic dissection were enrolled. According to number table, they were divided in to two groups randomly. The detection of Group A was under the condition of $120 \mathrm{kV}$ for tube voltage, 0 for iDOSE and iohexol $(350 \mathrm{mg} \mathrm{I} / \mathrm{mL})$ as contrast agent; while Group B was under the condition of $100 \mathrm{kV}$ for tube voltage, 3 for iDOSE and odixanol $(270 \mathrm{mg} \mathrm{I} / \mathrm{mL})$ as contrast agent. The image quality, radiation dose and iodine intakes were analyzed between two groups. Results There was no statistical significance between the two groups with subjective score of image quality and CT value. Group B reflected lower level for both radiation dose and iodine intake, compared to Group A , with statistical significance. Conclusion It deserves the clinical expansion of combined application of reducing the radiation dose by iDose low-dose scan strategy and using iodixanol as contrast agents in elderly CTA detection to aortic dissection, by effectively reducing the radiation dose and iodine intake.
\end{abstract}

\section{Introduction}

The rapid development of CT technology has brought a very useful diagnostic information. The aortic CT angiography (CTA) examination is the main imaging method for diagnosis of aortic dissection. it was announced by the German Federal Office[1], in 2003,CT examination had taken $6.1 \%$ proportion of all radiographic inspection, and the CT radiation dose could reached $51.9 \%$ in all radiographic inspection in Germany, and the data increased to $8 \%$ and $60 \%$ respectively in 2008. With the account of CT increased steadily, those data would get a further growth. According to reports [2], cancer patients can reach as high as $2 \%$ caused by the cumulative CT radiation.

Contrast agents are used to increase the concentration of iodine in the blood vessels to angiography definitely, which can provide more accurate information to diagnosis and treatment. But the application of contrast agent brought numerous negative impacts. Despite has the continuous improvement decreased acute side effects significantly; the long-term advice results of contrast agent such as liver and kidney damage have kept gradually increasing. Especially outstanding being, contrast-induced nephropathy (CIN) has been the third main causing of hospital acquired renal failure.[3] The elderly are particularly prone to adverse reactions to contrast agents due to their body function degraded by aging. It has become the main concern for both medical imaging community to reduce the dosage of CT and side-effect of contrast agents. However, there still has no relevant standard and quantitative standard for low radiation dose and iodine intake scanning technology in national and medical imaging specialty. How to decrease radiation dose and iodine intake on the basis of meeting the need of diagnosis and treatment has been a research hotspot. 
Reconstruction iterative (IR) is a new CT reconstruction technique, which is based on the statistical model of the noise, taking into account the actual geometrical size of the focus, the body and the detector. By establishing an accurate mathematical model for the generation and detection of X-ray, the algorithm recognizes and removes image noise selectively, decreases the noise and artifact to improve the image quality. In 2011, Philips Company took this technique in clinic, named as iDOSE, which performs IR into projective data space and imaging data space. This article combined the scan strategy of reducing the radiation dose by iDose low-dose and using iodixanol as contrast agents, the third generation of non-ionic iodine- developing agent, to explore the schedule of reducing radiation dose and iodine intake.

\section{Materials and Methods}

Clinical Subjects. 36 cases of elderly patients with aortic dissection were enrolled to the study, confirmed diagnosed in our hospital from May 2014 to Dec 2014, including 21males and 15 females. The age ranged from 63 to 85 , with the average age of $74+2.30$. According to the random number table, patients were divided into A and B groups, 18 cases in each group. The patients was exclude with any one of the conditions: hepatic or renal insufficiency, iodine allergy, coronary stent implantation and coronary artery bypass grafting

CT Scanning Methods. PHILPS 256 CT (iCT Brilliance, Royal PHILPS, Holland) was applied in this study. All patients underwent CT chest non-contrast scan firstly, with scan range from the thoracic inlet to the diaphragm level. Enhanced scan was followed non-contrast scan. The trigger point was set to the descending aorta, with the area as about $5 \mathrm{~mm} 2$ and trigger scan threshold as 90 $\mathrm{Hu}$. Aortic computed tomography angiography(CTA) scanned area was set from $50 \mathrm{~mm}$ above the aortic arch to the iliac artery. The non-ionic iodine contrast agent was bolus injected to the median vein of right elbow, in a velocity of $5.0 \mathrm{ml} / \mathrm{s}$, by double-syringe power injector (Urich, MEDTRON AG, Germany). Contrast agent injection standard was $80 \mathrm{ml}$ for $70 \mathrm{~kg}$ body weight, and dosage of contrast agent increased or decreased $5 \mathrm{ml}$ as the body weight increasing or decreasing $5 \mathrm{~kg}$. After all the contrast agents were injected, $25 \mathrm{ml}$ normal saline with concentration of $0.9 \%$ was continued to be injected in the rate of $5.0 \mathrm{ml} / \mathrm{s}$. The scan parameters were set as: automatic tube current, matrix of $512 \times 512$, collimator row of $128 \times 0.625 \mathrm{~mm}$, pitch of 0.758 , scan slice thickness of $10 \mathrm{~mm}$, reconstruction slice thickness of $1.5 \mathrm{~mm}$, reconstructing space of $1.5 \mathrm{~mm}$ minus. The tube voltages were set to $120 \mathrm{kV}$ for A group and $100 \mathrm{kV}$ for B group. Reconstruction parameters of iDose were set 0 for A group and 3 for B group. Iohexol $(350 \mathrm{mgI} / \mathrm{mL})$ and iodixanol $(270 \mathrm{mgI} / \mathrm{mL})$ were chosen as contrast agent for A, B group respectively.

Assessment Criteria. The images formatted by CT detection were uploaded to PHILPS PORTAL cloud workstation for post processing. The images of the 36 cases were assessed individually for photographic quality by two radiologists with at least associate senior professional title. Evaluation standard was classified 3 grades according to the image clarity of thoracic and abdominal aortas and their important branches. Grade 1 was marked 1point of score, and defined as poor image quality, unclear branch boundary, great noise, and unable to evaluated. Grade 2 was marked 2 points of score, and defined as average image quality, mild fuzzy branch boundary, low noise, able to assessed. Grade 3 was marked 3points of score, and defined as excellent quality, distinct branch boundary, slight noise and easy to evaluated.

Measuring CT Values of CTA Images. The CT values of CTA images were measured $50 \mathrm{~mm} 2$ of ascending aortas, arcus aortae, thoracic aortas, abdominal aortas, celiac trunk and iliac artery bifurcation respectively.

Calculation of the Radiation Dose. It was automatically calculated mean volume CT dose index (CTDIvol) and dose length product (DLP) by instrument after each scan. The effective dose (ED) was calculated according to DLP, which formula was $\mathrm{ED}=\mathrm{k} \times \mathrm{DLP}$ ( $\mathrm{k}$ valued as 0.017 recommended by commission of the European communities). 
Calculation of Iodine Intake. Iodine intake was count by $0.35 \times$ contrast agent volume $(\mathrm{ml})$ for Group A and by $0.25 \times$ contrast agent volume $(\mathrm{ml})$ for Group B.

Statistical Analysis.

SPSS18.0 statistical software was used for statistical analysis. All data were expressed as the mean \pm SD. Two independent sample t-test was used for calculating difference between the two groups of image quality, radiation dose and iodine intake. The difference was statistically significant as $\mathrm{P}<.05$.

\section{Results}

General information. The patients' general information was listed in Table 1. There was no significant difference in age, height and weight in both groups.

Table 1 Patients' General information of both A and B groups

\begin{tabular}{|c|c|c|c|c|}
\hline Items & Group A & Group B & t & P \\
\hline Age & $72 \pm 1.85$ & $75 \pm 2.41$ & 0.211 & 0.837 \\
\hline $\begin{array}{c}\text { Height } \\
(\mathrm{cm})\end{array}$ & $170 \pm 1.94$ & $169 \pm 2.05$ & 0.472 & 0.645 \\
\hline $\begin{array}{c}\text { Weight } \\
(\mathrm{kg})\end{array}$ & $81 \pm 11.23$ & $79 \pm 16.85$ & 1.495 & 0.151 \\
\hline
\end{tabular}

Score Comparison of Image Quality Between two Groups. There were 1, 1, 16 cases distributed respectively to $1,2,3$ points of image quality for group A, and the average score was $1.02 \pm 0.35$. While there were $0,1,17$ cases distributed respectively to $1,2,3$ points for group $\mathrm{B}$, and the average score was $0.99 \pm 0.54$. There was no statistical significance between the two groups with subjective score of image quality $(\mathrm{t}=1.638, \mathrm{P}>0.05)$.

CT Value Comparison of Aortic CTA Image between Two Groups. The CT value of aortic CTA image for both groups was listed in Table 2. The CT value was with no statistical difference in ascending aortas, arcus aortae, thoracic aortas, abdominal aortas, celiac trunk and iliac artery bifurcation $(\mathrm{P}>0.05)$.

TABLE 2 CT VALUE COMPARISON OF AORTIC CTA IMAGE BETWEEN TWO GROUPS $(\bar{x} \pm s, \mathrm{HU})$

\begin{tabular}{|c|c|c|c|c|}
\hline Body Parts & Group A & Group B & $\mathbf{t}$ & $\mathbf{p}$ \\
\hline Ascending aortas & $345.8 \pm 40.6$ & $331.4 \pm 51.2$ & 1.581 & 0.130 \\
\hline Ascending aortas & $315.7 \pm 21.3$ & $299.4 \pm 63.5$ & 1.414 & 0.174 \\
\hline Thoracic aortas & $355.6 \pm 28.6$ & $305.8 \pm 63.6$ & 1.607 & 0.125 \\
\hline Abdominal aortas & $303.6 \pm 31.7$ & $298.8 \pm 45.6$ & 1.607 & 0.125 \\
\hline Celiac trunk & $329.9 \pm 31.2$ & $338.6 \pm 23.5$ & 0.724 & 0.478 \\
\hline Iliac artery bifurcation & $298.7 \pm 26.1$ & $287.25 \pm 38.5$ & 0.142 & 0.888 \\
\hline
\end{tabular}

The Comparison of Radiation Dose and Iodine Intake between two Groups. The data of radiation dose and iodine intake for both groups was listed in Table 3. Group B reflected lower level for both radiation dose and iodine intake, compared to Group A, with statistical significance $(\mathrm{P}<$ $0.05)$. 
Table 3 The comparison of radiation dose and iodine intake between two groups

\begin{tabular}{|c|c|c|c|c|c|}
\hline Group & $\begin{array}{c}\text { CTDIvo } \\
\text { I (mGy) }\end{array}$ & $\begin{array}{c}\text { DLP } \\
(\mathbf{m G y} \cdot \mathbf{c m})\end{array}$ & $\begin{array}{c}\text { ED } \\
(\mathbf{m S v})\end{array}$ & $\begin{array}{c}\text { Dose of } \\
\text { contrast } \\
\text { agent(ml) }\end{array}$ & $\begin{array}{c}\text { Iodine } \\
\text { intake(g) }\end{array}$ \\
\hline $\mathrm{A}$ & $7.2 \pm 1.8$ & $\begin{array}{c}389.4 \pm \\
21.6\end{array}$ & $6.0 \pm 0.3$ & $80 \pm 2.33$ & $32.18 \pm$ \\
6.33 \\
\hline $\mathrm{B}$ & $3.1 \pm 0.9$ & $\begin{array}{c}199.4 \pm \\
33.5\end{array}$ & $1.9 \pm 0.8$ & $80 \pm 1.04$ & $\begin{array}{c}19.08 \pm \\
2.38\end{array}$ \\
\hline $\mathrm{t}$ & 3.365 & 2.998 & 4.032 & 0.001 & 5.726 \\
\hline $\mathrm{P}$ & $<0.05$ & $<0.05$ & $<0.05$ & 0.999 & $<0.05$ \\
\hline
\end{tabular}

\section{Discussions}

Recently, large range of data from arcus aortae to above symphysis pubis can be achieved as the continuous development of CT technology. In particular, Philips Brilliance iCT 256 can improve greatly the scan speed and shorten scan time, at the same time achieve the scan level to submillimeter. Therefore, the application of the 256 row CT in the diagnosis of aortic CTA not only can satisfy the diagnostic requirements, but also can reduce the time of examination. Even so, the radiation dose and iodine intake still need to be pay more attention to[4,5]. The main purpose of this study was to investigate the application value of the combined application of reducing the radiation dose by iDose low-dose scan strategy and using iodixanol $(270 \mathrm{mg} \mathrm{I} / \mathrm{mL})$ as contrast agents in elderly CTA detection to aortic dissection.

Risk of high radiation doses by CT has been the most important problem, which is worried by both radiologists and patients. There are a great deal methods to reduce the radiation doses received by patients, including dropping tube voltage or current in the process of CT scanning, increasing scan pitch, applying post-processing techniques such as adaptive iterative dose reduction(AIDR) or senior iterative reconstruction(iDOSE)[6,7]. Philips Brilliance iCT 256 can reduce scan time greatly because of its 128 rows of detectors, by which, 256 layers of images can be grabbed each circle. In this study, the method of reducing the radiation dose was directly dropping tube voltage when tube produced Xray, that turning down from $120 \mathrm{kV}$ of the instrument defaults to $100 \mathrm{kV}$. However, the quality of image was impaired with reduction of signal to noise ratio and spatial resolution by dropping tube voltage. In order to improving quality of image, iDose values were turned to 3 to process the image grabbed with $100 \mathrm{kV}$. It was not observed obvious differences from the results between both groups in subjective scoring and CT values in CTA images, while it was lower in radiation dose for Group B compared with Group A, which indicated iDose reconstruction technology could make up the loss of image quality caused by tube voltage reduction to meet the diagnostic requirements. [8,9]

Powerful effect produced by contrast agent provides CT scan more definite diagnostic information, which incomparable advantages general CT hardly can match. However, the safety problem of contrast agent has aroused great concern. With the development for decades, the contrast agent in the market has been greatly improved, even the permeability is even close to the plasma of contrast agent, but its side-effect is still inevitable. Especially CIN has been the main cause of Iatrogenic acquired renal injury or renal failure. As a special groups, the elder have a higher risk of CIN. Because in one hand ,they are generally with several risk factors like diabetes, metabolic disorders ,or hematangionosus, on the other hand, there kidney functions in a recession trend with age. Several researches[10] have proved the dose of contrast agent as an independent risk factor for CIN, which renal toxicity is proportional to the dose. On the basis of meeting the needs of the diagnosis and treatment, reduction of the contrast agent dosage can significantly decrease the morbidity of CIN. In this study, there was no significant difference between A and B two groups with contrast agent dose, because the dosage of contrast agent could not be insufficient to CTA image aorta in large range from the aortic arch to the iliac artery. As group B used iodixanol as contrast agent, which content of iodine 
was less $(270 \mathrm{mg} \mathrm{I} / \mathrm{mL})$, there were diffirences between group A and group B in the intake of iodine. The results showed group B kept the quality of image and satisfied the diagnostic need in the condition of decreasing iodine intake. For the elder, decreasing iodine intake in the process of CTA imaging, is beneficial to mitigating the damage to their renal function the renal function and reducing the incidence of CIN.

\section{Conclusions}

It deserves the clinical expansion of combined application of reducing the radiation dose by iDose low-dose scan strategy and using iodixanol (as contrast agents in elderly CTA detection to aortic dissection, by effectively reducing the radiation dose and iodine intake.

\section{Acknowledgment}

This study was supported by the fund of science and Technology Bureau of Qigihar.(NO. SFGG201306). The project name is "Study on aortic dissection type and image characteristics by CT256".

\section{References}

[1] J. Clerk Maxwell, A Treatise on Electricity and Magnetism, 3rd ed., vol. 2. Oxford: Clarendon, 1892, pp.68-73.

[2] Valentin J, International Commission on Radiation Managing patient dose in multi-detector computed Protection Tomography (MDCT). ICRP Publication 102[J]. Ann RP,2007,37(1):1-79.

[3] Rao QA, Newhouse JH. Risk of nephropathy after intravenous administration of contrast material: a critical literature analysis[J]. Radiology, 2006,239(2):392-7.

[4] Katzberg RW, Newhouse JH. Intravenous contrast medium-induced nephrotoxicity: is the medical risk really as great as we have come to believe?[J]. Radiology, 2010,256(1):21-8.R. Nicole,

[5] Zhao Y,Tao Z,Xu Z . Toxic effects of a high dose of non-ionic iodinated contrast media on renal glomerular and aortic endo-thelial cells in aged rats in vivo[J]. Toxicology Letters,2011,202(3):253-260

[6] Giulia R, Carlo B, Cristina Q, et al. Contrast agents and renal cell apoptosis. Eur Heart J,2008,29:2569-2576

[7] KocG, Courtier JL, Phelps A, et al. Computed tomography depiction of small pediatric vessels with model-based iterative reconstructmn[J]. Pediatr Radio1,2014,44(7):787-794

[8] Ghafourian K,Younes D,Simprini L A,et al.Scout view X-ray attenuation versus weight-based selection of reduced peak tube voltage in cardiac CT angiography[J].JACC Cardiovasc Imaging,2012,5(6):589-595.

[9] Marenzi G,Assanelli E,Campodonico J,et al.Contrast volume during primary percutaneous coronary intervention and subsequent contras-induced nephropathy and mortality[J].Ann Intern Med,2009,150(3):170-177.

[10] Gordic S, Morsbach F,Schmidt B,et al. Ultralow-dose chest computed tomography for pulmonary nodule detection;first performance evaluation of single energy scanning with spectral shaping[J].Invest Radio1,2014,49(7):465-473.. 